\title{
Antioxidants in health and disease
}

\author{
Ginter $E^{1}$, Simko $V^{2}$, Panakova $\mathrm{V}^{1}$ \\ Slovak Medical University, Bratislava, Slovakia. ginter.emil@mail.t-com.sk
}

\begin{abstract}
Research on antioxidants proceeds at full speed after a partial decline of public interest, when claims on effectiveness of mega doses of vitamin $\mathrm{C}$ proved unfounded. The main role of antioxidants is to liquidate the uncontrolled production of reactive oxygen species (ROS) that is being linked to pathogenesis of cardiovascular disease (CVD), malignancy, diabetes type 2, mechanism of infection, fibrogenesis and some neurological disorders. This review summarizes the most recent reports on antioxidants, published since 2010. Follow up data on vitamins $C$ and $E$ focus on their potential for immune modulation and on endothelial nitric oxide bioavailability. Previously well established antioxidants carotenoids and polyphenols are still much in scientific interest. Interestingly, several antioxidants (for example rasveratrol and feruloylnoradrenaline) are generated in infected or injured plants. An intensive attention is directed to resveratrol. This compound, in addition to antioxidation, stimulates nitric oxid production, protects endothelial cells from oxidative functional damage, lowers platelet aggregation and directly inhibits cyclic adenosine monophosphate-specific phosphodiesterases. Recently discovered feruloylnoradrenaline in microbially infected tomatoes is reported to have 14 times the antioxidant power of resveratrol. With all this new information, it is important to point out the prevailing opinion that additional supplements of antioxidants are not needed, as long as the organism has adequate stores of antioxidants and the diet is normal in composition and in quantity (Fig. 3, Ref. 34). Text in PDF www.elis.sk.

Key words: reactive oxygen species (ROS), antioxidants, vitamin C and E, carotenoids, polyphenols, immune modulation, nitric oxide (NO), bioavailability, resveratrol, feruloylnoradrenaline.
\end{abstract}

Antioxidant molecules prevent oxidation of body metabolites. Oxidation promotes transfer of electrons or hydrogen to oxidants, predominantly to oxygen. Oxidation generates free radicals. These jump-start a chain of events that compromise the cells. Antioxidants block these chain reactions by removing the reactive oxygen species (ROS). ROS are believed to cause or aggravate several human pathologic processes such as heart disease, stroke, cancer, neurodegenerative diseases and many other disorders. As part of their adaptation from marine life, terrestrial plants began producing antioxidants such as ascorbic acid, polyphenols, tocopherols and innumerable other antioxidants. Research in antioxidants produced a multitude of reports that are too complex for a simple review. Submission of a query "antioxidants" to Pubmed from 2010 to early 2013, resulted in a return of over 50,000 articles published in referenced journals. The purpose of this review is to bring attention to very recent reports on biologically effective antioxidants, published after 2010 . Only briefly will be introduced the well established antioxidants, vitamins $\mathrm{C}$ and $\mathrm{E}$, glutathione and selenium

\section{Antioxidants well established and widely used}

Vitamin $E$ is the collective name for a set of eight related tocopherols and tocotrienols. Tocopherols are fat-soluble vitamins

${ }^{1}$ Slovak Medical University, Bratislava, Slovakia, emeritus ${ }^{2}$ State University New York, Downstate Medical Center at Brooklyn, USA

Address for correspondence: E. Ginter, RND, DSc, Racianska 17, SK83102 Bratislava, Slovakia. with antioxidant properties. Of these, the $\alpha$-tocopherol that blocks the ROS and prevents generation of oxidized low-density lipoproteins (LDL-ox) was initially considered a promising antioxidant. LDL-ox and ROS may directly cause endothelial dysfunction by reducing endothelial nitric oxide (NO) bioavailability $(1,2)$. It was expected that vitamin E can to some degree prevent atherogenesis but several large, randomized controlled trials have failed to confirm the benefits of vitamin E in CVD prevention (3). Recommended daily intake of vitamin E is $10-15 \mathrm{mg}$. Suggestions to use substantially higher intake of vitamin $\mathrm{E}$ and a prolonged use have not yet been recommended in human beings.

Vitamin C-ascorbic acid is redox catalyst found in both animals and plants. As the synthesis of ascorbic acid has been lost by mutation during primate evolution, humans must obtain it from the diet. Most other animals are able to produce vitamin C. Ascorbic acid is required for the conversion of the pro collagen to collagen. It is maintained in its reduced form by reaction with glutathione. Interest in vitamin $\mathrm{C}$ shares similarity with vitamin $\mathrm{E}$. Initial overly enthusiastic reports on anti-atherogenic and anti-cancerogenic benefit of mega doses of vitamin $\mathrm{C}$ were not confirmed. However, it is essential to prevent chronic vitamin $\mathrm{C}$ deficiency that may be a factor in increased mortality (4) and contribute to atherogenesis. Ascorbic acid has a broad beneficial impact on body physiology including oxidative stress suppression and immune modulation (5), it also affects the blood pressure (6). Daily dose of 100-200 $\mathrm{mg}$ per day is the optimum dietary intake of vitamin $\mathrm{C}$ for the majority of the adult population, maximizing the vitamin's potential 
603-606

health benefits (7). More information on vitamin $\mathrm{C}$ is to be found in a recent detailed review (8).

Glutathione (GSH) is a tripeptide synthesized from glutamate, cysteine and glycine, by $\gamma$-glutamylcysteine synthetase and glutathione synthetase. It efficiently scavenges ROS and free radicals preventing an increase in the oxidative stress process. It has an important role in regeneration of oxidized ascorbic acid. Glutathione is constantly synthesized in the body: that explains why a trial with glutathione supplementation in healthy adults (9) did not produce significant improvement in biomarkers of oxidative stress and in the glutathione body reserve.

Selenium. For many years, it was regarded as a toxic element, causing numerous diseases but research in the past 50 years has revealed its beneficial role, especially as a component of selenoproteins (10). Exogenous selenium intake may benefit people with low tissue stores. However, those with an adequate-to-high status might be affected adversely and should not take selenium supplements. Additional information on selenium is in an excellent recent review (11).

Uric acid is the antioxidant with the highest concentration in human blood. It is produced from xanthine. The role of uric acid in prevention of chronic disease has not been established. Hyperuricemia is an independent risk factor of nephropathy. There are adequate body stores of uric acid and it is not used for exogenous intake.

\section{Novel antioxidants: A perspective}

Carotenoids and polyphenols are well established antioxidants and their new very effective derivatives have been reported. These are distinguished by substantial structure difference (Fig. 1). Carotenoids are typical with a long polyunsaturated carbohydrate molecule, alternating simple and duplicate bonds. Polyphenols are a group of chemical substances characterized by the presence of more than one phenol unit.

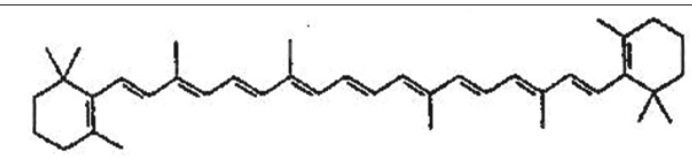

\section{Carotenoid (Beta-carotene)}

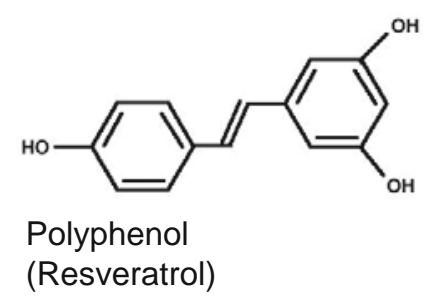

Fig. 1. Different structure of carotenoids and polyphenols.

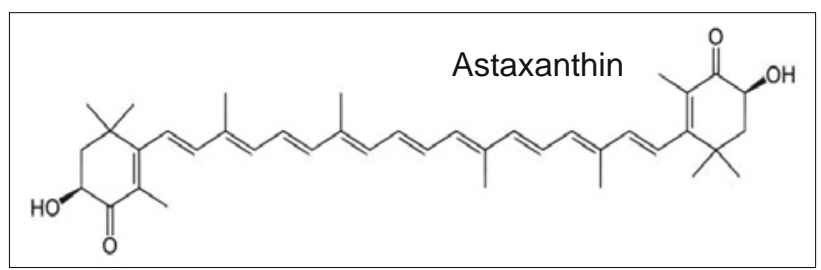

Fig. 2. Unusual structure of astaxanthin (on both ends of the carbohydrate a hydroxy- and keto-group).

The main source of polyphenols is fruit and vegetable, from animal products it is the egg yolk.

\section{Carotenoids}

The best known of these compounds is beta carotene, a precursor of vitamin A. Its protective function includes both quenching of singlet oxygen and of damaging free radicals. Initial expectation that beta carotene would prevent malignancy and CVD did not materialize $(3,12)$. There is a recent comprehensive review on beta carotene (13).

Another carotenoid, lycopene is also a very efficient oxygen and free radical quencher and is the prime carotenoid in plasma and other tissues. In addition, lycopene is involved in a direct modulation of redox signalling. ROS are known to act as second messengers in the modulation of cellular signalling, leading to gene expression changes. Lycopene may control redox-sensitive molecular targets, affecting enzyme activities and expressions and modulating the activation of transcription factors. Despite these promising reports, it is difficult at this time to directly relate available experimental data on lycopene to human pathophysiology (14). There surfaced a report (15) linking high blood level of lycopene with cerebrovascular accidents and ischemic stroke.

Lutein is a xanthophyll and one of 600 known naturally occurring carotenoids. Lutein is a potent antioxidant that neutralizes ROS. After absorption from the intestine, lutein accumulates in the eye retina and lens. Recent reports claim that lutein slows degenerative retinal processes, macular degeneration, lens cataracts and progression of colorectal malignancy (16-19). Similar effect has been claimed for astaxanthin (20-22). This compound differs from other carotenoids: its molecular structure has on both ends of the carbohydrate a hydroxy- and keto-group (Fig. 2).

\section{Polyphenols}

Polyphenols are the group of chemical substances found in plants, characterized by the presence of more than one phenol unit. The largest and best studied polyphenols are the flavonoids, which include several thousand compounds (23). Red wine is among the richest sources of polyphenols $(24,25)$. Of all polyphenolic compounds in red wine, most attention has been focused on resveratrol. This compound is produced in the skin of red grapes by the effect of fungi and bacteria. Experimental studies have provided evidence that resveratrol possesses antioxidant, antiapoptotic, antiangiogenic and antidiabetic effects. Exposure to resveratrol stimulates nitric oxid (NO) production, protects endothelial cells from oxidative functional damage and also affects the antiplatelet activity 
and mononucleocyte adhesion (26). In humans, resveratrol, at the concentrations produced in plasma by moderate intake of wine, stimulated NO production in platelets and it lowered platelet aggregation. Despite scepticism concerning the biological availability of resveratrol, a growing body of in vivo evidence indicates that resveratrol has protective effects in several stress and disease models. Resveratrol may hold promise in the management of myocardial ischemia and ox-LDL-hypercholesterolemia (27). Neuroprotective effects of resveratrol have been found in models of neurodegeneration (Alzheimer's, Parkinson's or Huntington's disease), of CNS ischaemia and in models of brain and spinal cord injury. Further clinical data are still needed to confirm these reports (28). Resveratrol seems to be well tolerated and no marked toxicity was reported. Recent findings are encouraging and provide strong motivation to perform additional clinical studies to confirm the therapeutic value of resveratrol. There is more additional information in a recent extensive review on resveratrol (29). Studies in vitro show that resveratrol enhances insulin-stimulated glucose uptake in skeletal muscle, liver, and adipocytes and stimulates insulin secretion via inhibition of $\beta$-cell $\mathrm{K}_{\text {ATP }}$ channels These observations have been confirmed in vivo in several animal models, including aging, dietinduced obesity, and diabetic mice. Importantly, the key metabolic effects of resveratrol can be monitored in relevant tissues (muscle, fat, and liver), thus providing critical insight into mechanisms. These effects include increased mitochondrial biogenesis and oxidative phosphorylation, increased SIRT1 (intracellular regulatory protein with mono-ADP-ribosyltransferase activity), AMP kinase and PGC- $1 \alpha$ activation (a regulator of mitochondrial biogenesis and function) and decreased inflammatory markers in tissues.

\section{The most recent data on antioxidants}

Interestingly, several antioxidants originate in compromised or infected plants. Resveratrol is produced in the skin of red grape under the influence of mold and bacteria. Based on this discovery, a novel antioxidant feruloylnoradrenaline was isolated from tomatoes infected by microbial pathogens (30). This new phytochemical is reported to have 14 times the antioxidant power of resveratrol. Because of its high antioxidant properties, it may have a potential for the pharmaceutical industry, to develop products for management disorders such as cancer and heart disease. Critical clinical studies are necessary and forthcoming.

Procyanidins isolated from grape seeds represent dipeptidylpeptidase 4 (DPP4) inhibitors which are among the novel treatment in type 2 diabetes (31).

\section{Conclusion}

The main role of antioxidants is to liquidate reactive oxygen species (ROS) that occur in three main forms: superoxide anion, peroxide (hydrogen peroxide) and hydroxyl radical. Their unpaired electron is responsible for a high biological activity. Yet, it would be inaccurate to assign to ROS only a damaging biological effect. ROS are generated in a number of reactions essential to life, as in apoptosis (programmed cell death). Phagocytic cells generate

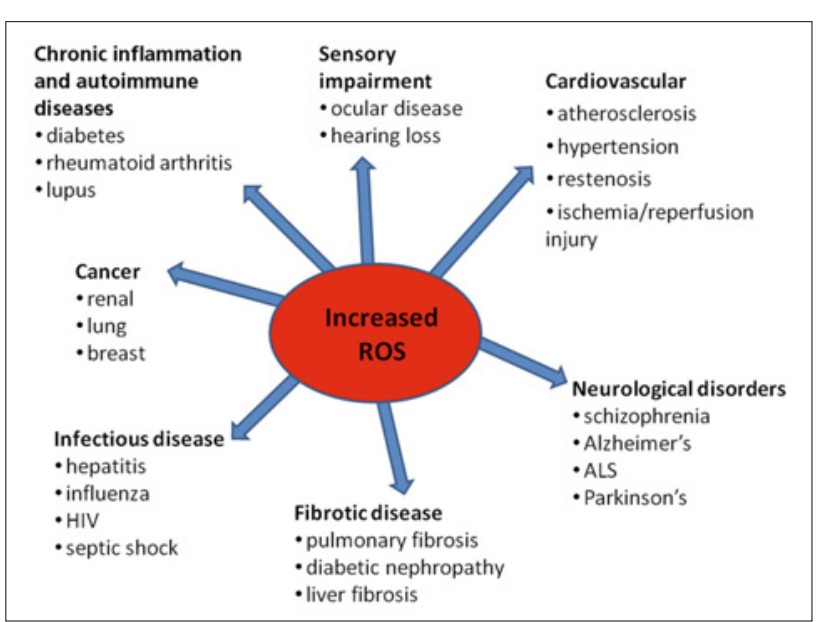

Fig. 3. Great spectrum of pathological effects of reactive oxygen species (According to Brieger et al (33)).

ROS to kill invading pathogens. ROS are involved in intercellular and intracellular signalling. In the heart, redox signalling regulates several physiological processes (e.g. excitation-contraction coupling) and it is involved in a wide variety of pathophysiological and homoeostatic or stress- response pathways (32). ROS also act as mitogens. Addition of superoxide or hydrogen peroxide to a variety of cultured cells leads to an increased rate of DNA replication and cell proliferation. Thus, excess accumulation of ROS induces a chain of pathological events. Such complex interaction of ROS is documented in Figure 3 (33). Excess of ROS induces a series of pathological processes that may promote the risk of CVD, malignancy, diabetes type 2, fibrogenesis and some neurological disorders. Antioxidants reduce such risk but without impairment of signalling by ROS (34).

At present it has not been decisively determined what type of antioxidants and at what dose may be beneficial. There is concern that a long term intake of high dose of a single antioxidant may impair the biological integrity of the body. Such supplements may do more harm than good. An inappropriate selection of antioxidants or indiscriminate dosing may induce side effects. Prevailing cautious advice indicates that as long as body has adequate levels of antioxidants and the dietary intake is normal, additional supplements are not needed. Without any doubt fruit, vegetables, nuts and plant oils, especially the olive oil are indeed good for health and may help in prevention of heart disease, some types of malignancy and age related disorders.

\section{References}

1. Traber MG, Stevens JF. Vitamins C and E: beneficial effects from a mechanistic perspective. Free Radic Biol Med 2011; 51 (5): 1000-1013.

2. Wong RS, Radhakrishnan AK. Tocotrienol research: past into present. Nutr Rev 2012; 70 (9): 483-490.

3. Riccioni G, D’Orazio N, Salvatore C et al. Carotenoids and vitamins $\mathrm{C}$ and $\mathrm{E}$ in the prevention of cardiovascular disease. Int J Vitam Nutr Res 2012; 82 (1): 15-26. 
4. Dashti-Khavidaki S, Talasaz AH, Tabeefar H et al. Plasma vitamin C concentrations in patients on routine hemodialysis and its relationship to patients' morbidity and mortality. Int J Vitam Nutr Res 2011; 81 (4): 197-203.

5. Pohanka M, Pejchal J, Snopkova S et al. Ascorbic acid: an old player with a broad impact on body physiology including oxidative stress suppression and immunomodulation: a review. Mini Rev Med Chem 2012; 12 (1): 35-43.

6. Juraschek SP, Guallar E, Appel LJ et al. Effects of vitamin C supplementation on blood pressure: a meta-analysis of randomized controlled trials. Am J Clin Nutr 2012; 95 (5): 1079-1088.

7. Frei B, Birlouez-Aragon I, Lykkesfeldt J. Authors' perspective: What is the optimum intake of vitamin C in humans? Crit Rev Food Sci Nutr 2012; 52 (9): 815-829.

8. Du J, Cullen JJ, Buettner GR. Ascorbic acid: chemistry, biology and the treatment of cancer. Biochim Biophys Acta 2012; 1826 (2): 443-457.

9. Allen J, Bradley RD. Effects of oral glutathione supplementation on systemic oxidative stress biomarkers in human volunteers. J Altern Complement Med 2011; 17 (9): 827-833.

10. Bodnar M, Konieczka P, Namiesnik J. The properties, functions, and use of selenium compounds in living organisms. J Environ Sci Health C Environ Carcinog Ecotoxicol Rev 2012; 30 (3): 225-252.

11. Rayman MP. Selenium and human health. Lancet 2012; 379:12561268.

12. Aune D, Chan DS, Vieira AR et al. Dietary compared with blood concentrations of carotenoids and breast cancer risk: a systematic review and meta-analysis of prospective studies. Am J Clin Nutr 2012; 96 (2): 356-373.

13. Böhm F, Edge R, Truscott TG. Interactions of dietary carotenoids with singlet oxygen (1O2) and free radicals: potential effects for human health. Acta Biochim Pol 2012; 59 (1): 27-30

14. Palozza P, Catalano A, Simone R et al. Lycopene as a guardian of redox signalling. Acta Biochim Pol 2012; 59 (1): 21-25.

15. Karppi J, Laukkanen JA, Sivenius J et al. Serum lycopene decreases the risk of stroke in men. A population-based follow-up study. Neurology 2012; 79: 1540-1547.

16. Murray IJ, Makridaki M, van der Veen RL et al. Lutein supplementation over a one year period in early AMD might have a mild beneficial effect on visual acuity; the CLEAR study. Invest Ophthalmol Vis Sci 2013 Feb 5 .

17. Okuyama Y, Ozasa K, Oki K et al. Inverse associations between serum concentrations of zeaxanthin and other carotenoids and colorectal neoplasm in Japanese. Int J Clin Oncol 2013 Feb 5.

18. Jung $\mathrm{S}$, Wu K, Giovannucci $\mathbf{E}$ et al. Carotenoid intake and risk of colorectal adenomas in a cohort of male health professionals. Cancer Causes Control 2013 Feb 1.
19. Yao Y, Qiu QH, Wu XW et al. Lutein supplementation improves visual performance in Chinese drivers: 1-year randomized, double-blind, placebocontrolled study. Nutrition. 2013 Jan 26. pii: S0899-9007 (12)00428-5. doi10.1016/j.nut.2012.10.017.

20. Kidd P. Astaxanthin, cell membrane nutrient with diverse clinical benefits and anti-aging potential. Altern Med Rev 2011; 16 (4): 355-364.

21. Kim JH, Chang MJ, Choi HD et al. Protective effects of Haematococcus astaxanthin on oxidative stress in healthy smokers. J Med Food 2011; 14 (11): 1469-1475.

22. Nakagawa K, Kiko T, Miyazawa T et al. Antioxidant effect of astaxanthin on phospholipid peroxidation in human erythrocytes. Br J Nutr 2011; 105 (11): 1563-1571.

23. Ginter E, Simko V. Plant polyphenols in prevention of heart disease. Bratisl Lek Listy 2012; 113 (8): 476-480.

24. Neveu V, Perez-Jiménez J, Vos F et al. Phenol-Explorer: an online comprehensive database on polyphenol contents in foods. Database (Oxford). 2010; 2010:bap024.

25. Arranz S, Chiva-Blanch G, Valderas-Martínez $P$ et al. Wine, beer, alcohol and polyphenols on cardiovascular disease and cancer. Nutrients 2012; 4 (7): 759-781.

26. Voloshyna I, Hussaini SM, Reiss AB. Resveratrol in cholesterol metabolism and atherosclerosis. J Med Food. 2012; 15 (9): 763-773.

27. Nakata R, Takahashi S, Inoue $\mathbf{H}$. Recent advances in the study on resveratrol. Biol Pharm Bull 2012; 35 (3): 273-279.

28. López-Miranda V, Soto-Montenegro ML, Vera G et al. Resveratrol: a neuroprotective polyphenol in the Mediterranean diet. Rev Neurol 2012; 54 (6): 349-356.

29. Nakata R, Takahashi S, Inoue H. Recent advances in the study on resveratrol. Biol Pharm Bull 2012; 35 (3): 273-279.

30. López-Gresa MP, Torres C, Laura Campos $L$ et al. Identification of defence metabolites in tomato plants infected by the bacterial pathogen Pseudomonas syringae. Environm Exp Botany 2011; 74: 216-228.

31. González-Abuín N, Martínez-Micaelo N, Blay M et al. Grape seedderived procyanidins decrease dipeptidyl-peptidase 4 activity and expression. J Agric Food Chem 2012; 60 (36): 9055-9061.

32. Burgoyne JR, Mongue-Din H, Eaton $P$ et al. Redox signaling in cardiac physiology and pathology. Circulat Res 2012; 111 (8): 1091-1106.

33. Brieger K, Schiavone S, Miller FJ et al. Reactive oxygen species: from health to disease. Swiss Med Wkly 2012; 142: w13659.

34. Niki E. Do antioxidants impair signaling by reactive oxygen species and lipid oxidation products? FEBS Lett 2012; 586 (21): 3767-3770.

Received March 27, 2013. Accepted May 1, 2014. 